1 ORIGINAL ARTCLE

\title{
Comparison of the Efficacy of Levothyroxine Suppression Dose Computed Based on Actual Body Weight vs. Lean Body Mass among Differentiated Thyroid Cancer Patients: A Randomized Controlled Trial
}

\author{
Johanna Lu, Reynaldo Rosales, Oliver Allan Dampil \\ Section of Endocrinology, Diabetes and Metabolism, Department of Medicine, St. Luke's Medical Center, Quezon City, Philippines
}

\begin{abstract}
Background. The dose of levothyroxine (LT4) after total thyroidectomy is usually computed based on actual body weight. However, metabolism through deiodination of thyroid hormones usually occur in the lean body compartment. An optimal dose to reduce delay in achieving target levels is essential to improve quality of life, reduce risk factors and cost.

Objectives. Comparison of the efficacy of two methods of computation for the initial levothyroxine dose in patients with differentiated thyroid cancer based on actual body weight vs. lean body mass in achieving thyroid-stimulating hormone (TSH) goals.

Methodology. Randomized, single-center, 12-week open label controlled trial among adult patients with differentiated thyroid cancer post total thyroidectomy who underwent radioactive therapy at St. Luke's Medical Center Quezon City from July-December 2018. Participants were divided into 2 groups - Actual Body Weight (ABW) and Lean Body Mass (LBM). Levothyroxine dose was computed based on ABW vs. LBM and TSH determined at $6^{\text {th }}$ and $12^{\text {th }}$ weeks after.

Results. 52 participants (ABW $n=26$; LBM $n=26$ ) were included. ABW group had significantly higher mean LT4 dosage $(2.2 \mathrm{mcg} / \mathrm{kg})$ compared to the LBM group $(1.4 \mathrm{mcg} / \mathrm{kg})(p$-value $<0.001)$. ABW group had lower TSH levels at 6 th week $(5.7 \mathrm{ulU} / \mathrm{mL})$ than LBM group $(18.4 \mathrm{ulU} / \mathrm{mL})$ but the difference was not significant. ( $p$-value=0.064). A significantly lower TSH level was observed at week 12 in the ABW group $(1.6 \mathrm{ulU} / \mathrm{mL})$ compared to the LBM group $(3.8 \mathrm{ulU} / \mathrm{mL})$ $(p$-value $=0.010)$. However, both methods were not associated with achievement of TSH goal at $6^{\text {th }}$ and $12^{\text {th }}$ week $(p$-value $=0.512$ and 0.780 , respectively).

Conclusion. Among patients with differentiated thyroid cancer who underwent 1st time RAI therapy, ABW method of computation for LT4 dosage is better compared to the LBM method due to the lower TSH trend seen at $6^{\text {th }}$ week and statistically significantly lower mean TSH at week 12, although, both method of computations did not achieve target TSH levels at the $6^{\text {th }}$ nor $12^{\text {th }}$ week.
\end{abstract}

Key words: lean body mass, differentiated thyroid cancer, TSH suppression

\section{INTRODUCTION}

Surgical removal of the thyroid gland requires replacement with exogenous thyroid hormone medication. Patients with differentiated thyroid cancer should be treated with thyroid hormone after total thyroidectomy. The purpose of the replacement is to correct surgeryinduced hypothyroidism and to suppress the pituitary secretion of TSH which retards the growth and spread of the neoplastic disease. ${ }^{1}$ This concept of tumor growth accelerated by TSH is well established in the clinical and experimental literature. ${ }^{2}$

According to surgical literature, the time to achieve euthyroidism following thyroidectomy ranges from 2 weeks to 2.5 years, with a median of 3.6 months and is dependent on the magnitude of change in dose from baseline. ${ }^{3}$
Based on body weight, hypothyroid patients with minimal endogenous thyroid function require LT4 doses of 1.6 to $1.8 \mathrm{mcg} / \mathrm{kg}$ of ABW while LT4 doses in thyroid cancer patients requiring TSH suppression are generally higher ranging from 2.1 to $2.7 \mathrm{mcg} / \mathrm{kg}$ of $\mathrm{ABW} .{ }^{4}$ Evidence shows that ABW, TSH goal, ideal body weight, etiology of hypothyroidism, degree of serum TSH elevation, pregnancy, and age can influence dose requirement. ${ }^{4}$ While some studies regarding primary hypothyroidism have shown that the LT4 requirement is more closely related to LBM than ABW, the drawback is that accurate assessment of LBM requires complex techniques and instrumentation such as body impedance measurements and dual energy X-ray absorptiometry (DEXA) which are not routinely available in clinical practice, and may be tedious and not cost-effective. ${ }^{5}$ Still, for newly diagnosed patients with hypothyroidism, it is difficult to predict the final dose each
ISSN 0857-1074 (Print) | eISSN 2308-118x (Online) Printed in the Philippines

Copyright (C) 2019 by the JAFES

Received: June 20, 2019. Accepted: August 14, 2019.

Published online first: November 10, 2019.

https://doi.org/10.15605/jafes.034.02.06
Corresponding author: Johanna M. Lu, MD

Section of Endocrinology, Diabetes and Metabolism

Department of Medicine, St. Luke's Medical Center, Quezon City

279 E. Rodriguez Sr. Avenue, Quezon City, Philippines 1112

Telefax: +632-8723-0101 local 5210

E-mail:wpwphanna@yahoo.com

ORCiD: https://orcid.org/0000-0002-9084-8798 
patient will require. Often, in other countries, the dose is started empirically and adjustments are made based on the TSH results.

Many reviews report a period of 4 to 6 months before normalization of TSH levels is attained. ${ }^{4}$ In line with this, as the number of patients requiring thyroid hormone replacement increases, a more rapid normalization could be beneficial to this group in reducing cardiac risk factors, improvement of quality of life, reducing overall cost, and limiting the difficulty of repeated testing, which compels multiple visits to the hospital for repeated TSH testing.

A few studies ${ }^{1-4}$ have reported that LBM and body mass index (BMI) are better predictors of LT4 dose than ABW in achieving TSH goals among hypothyroid patients, but their results have been conflicting. Although the guidelines recommended a range for starting LT4 replacement in thyroid cancer, it did not recommend what dose to start in low, intermediate, or high risk differentiated thyroid cancer. In this study, we aim to compare the efficacy of two different methods of computation for the initial levothyroxine dose in patients diagnosed with differentiated thyroid cancer based on ABW vs. LBM in achieving target TSH suppression goals.

\section{METHODOLOGY}

\section{Study design}

We performed a randomized, 12-week open label controlled trial among adult patients diagnosed with differentiated thyroid cancer who underwent first time Radioactive (RAI) therapy at St. Luke's Medical Center, Quezon City from July- December 2018.

\section{Inclusion criteria}

Adult (18 to 65 years old) patients diagnosed with differentiated thyroid cancer who underwent $1^{\text {st }}$ time RAI therapy after total thyroidectomy were included in the study.

\section{Exclusion criteria}

Patients taking medications known to interfere with levothyroxine absorption or alter levothyroxine binding proteins (e.g., estrogen and testosterone, iodine, propranolol, amiodarone, lithium, dopamine agonists or antagonists, somatostatin analogues, steroids, phenytoin, carbamazepine, sertraline, rifampin, bile acid sequestrants, antacids, kayexalate, cholestyramine, colestipol, and raloxifene), pregnant or lactating patients, patients with chronic, serious diseases such as cardiac, pulmonary, gastro-intestinal, renal, and pituitary diseases were excluded in the study.

\section{Study procedure}

Adult patients diagnosed with differentiated thyroid cancer admitted for first dose of RAI therapy at the St. Luke's Medical Center Quezon City Social Service as well as those seen privately by participating endocrinologists were recruited in the study. Informed consent was obtained prior to enrollment. A total of 62 patients met the criteria and were included in this study. Five participants were excluded due to poor compliance and 5 participants were lost to follow up. The remaining 52 participants were included in the study and were randomized to ABW and LBM groups via a web based generated random numbers assigned by the primary investigator. Risk stratification for disease recurrence and or persistence was done based on the 2015 ATA Guidelines for Adult Patients with Thyroid Nodules and Differentiated Thyroid Cancer. Thirteen patients were stratified under intermediate risk and 39 patients were classified low risk. Baseline demographics which included age, weight and height, BMI, medications taken, year diagnosed with thyroid cancer, date of surgery and current medical conditions were obtained from the participants. Weight and height were taken upon admission using calibrated weighing scale at the nurses' station. Standard protocol in measuring height and weight was followed. LBM was computed based on validated anthropometric prediction equations for estimation of lean body mass in Indian men and women ${ }^{6}$ as follows:

For Men: $\mathrm{LBM}=-15.605-(0.032 \times \mathrm{A})+(0.192 \times \mathrm{H})+(0.502 \times \mathrm{W})$ ForWomen: LBM=-13.034- $(0.018 \times \mathrm{A})+(0.165 \times \mathrm{H})+(0.409 \times \mathrm{W})$ (where $A$ is age in years, $W$ is body weight in $\mathrm{kg}$ and $H$ is body height in $\mathrm{cm}$ )

Dose of levothyroxine was computed based on the formula: Actual Body Weight: Levothyroxine dose = ABW in $\mathrm{kg} \times 2.2$ Lean Body Weight: Levothyroxine dose = LBM in $\mathrm{kg} \times 2.2$

The participants were instructed to take LT4 on an empty stomach, to wait one hour prior to eating, to separate thyroid hormone from other medications by four hours and to take LT4 if possible, about the same time every day. The participants were called every week to remind them about compliance. All participants were prescribed with the same name brand thyroid hormone and were maintained on the same LT4 preparation. The computed dose of LT4 was then rounded off to the nearest available preparation ( $25 \mathrm{mcg}$, $50 \mathrm{mcg}$, $100 \mathrm{mcg}, 150 \mathrm{mcg}$ ).

Participants in both groups were asked to follow up with their attending physician at $6^{\text {th }}$ and $12^{\text {th }}$ weeks wherein TSH values were obtained (2 determinations). Among participants who did not achieve TSH suppression goals at the initial determination, a repeat TSH was obtained after 6 weeks and LT4 dose was adjusted with increments or decrements of $25 \mathrm{mcg}$.

The primary outcome was mean TSH levels between ABW and LBM groups at $6^{\text {th }}$ and $12^{\text {th }}$ weeks post RAI while the secondary outcome was achievement of TSH suppression goal defined as proportion of patients achieving adequate suppression (TSH of 0.5 to $2 \mathrm{uIU} / \mathrm{mL}$ for low risk and TSH 0.1 to $0.5 \mathrm{uIU} / \mathrm{mL}$ for Intermediate Risk) at $6^{\text {th }}$ and $12^{\text {th }}$ weeks post RAI.

Sample size was calculated based on the comparison between mean TSH level 6 weeks after treatment between patients given LT4 calculated using ABW versus LBM. Assuming that mean TSH level for dose given LT4 using $\mathrm{ABW}$ is $2.25 \pm 0.91 \mathrm{SD}$, and those given LT4 using LBM, $3.27 \pm 1.097,7$ with an alpha error of 5 percent power of $95 \%$ and a two tailed alternative hypothesis, sample size required was 26 per group or 52 per 2 groups. 


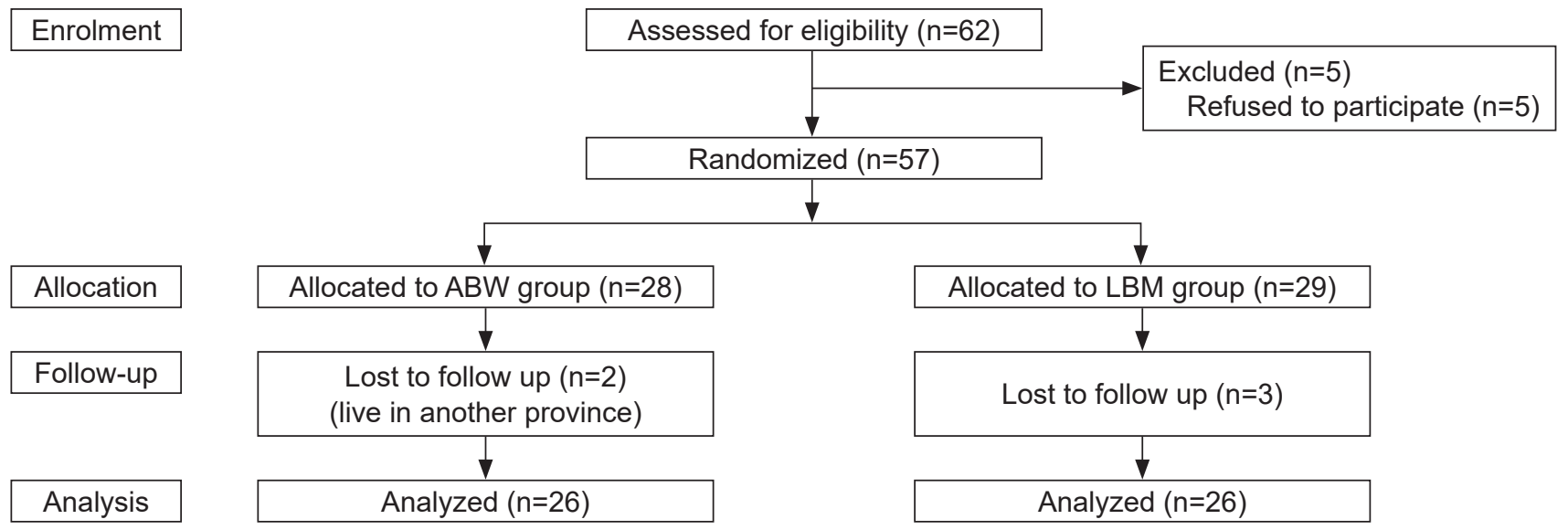

Figure 1. Study participants: Enrollment, allocation, follow-up and analysis.

\section{Data analysis}

Descriptive analysis of the demographic and clinical profile of patients with differentiated thyroid cancer was done. Continuous variables were described using mean and standard deviation. Categorical variables were described using frequency and percentage. Wilcoxon ranksum test was used to compare non-normal continuous variables (age, BMI, lean body mass, LT4 dose, TSH levels) between $\mathrm{ABW}$ and LBM groups. Chi-square test was done to compare categorical variables (sex and achievement of TSH targets). The association between method of calculation and TSH levels during $6^{\text {th }}$ and $12^{\text {th }}$ weeks was assessed using simple linear regression. The odds ratio and the corresponding 95\% confidence interval for the achievement of the suppression goal was also calculated using simple logistic regression. A p-value of less than 0.05 is statistically significant. STATA 14 SE version was used in all statistical analysis.

\section{Ethical considerations}

This study was conducted in accordance with the ethical principles (Declaration of Helsinki and the National Guidelines for Biomedical Research of the National Ethics Committee) of the Philippines. The clinical protocol and all relevant documents was reviewed and approved by the St. Luke's Medical Center Quezon City Institutional Ethics Review Committee (IERC). Patient confidentiality was respected by ensuring anonymity of patient.

Patient information and records of the procedure were kept strictly confidential by the primary investigator. The source documents were coded and did not contain identifying information in order to ensure confidentiality.

The source documents are kept by the primary investigator in a secure location in the research library of the section and after 5 years of the study, hard copy of the data will be shredded.

Written documentation of informed consent was provided during recruitment of patients by the primary investigator prior to obtaining data. Participation in the study was purely voluntary and without financial compensation. Recruitment of the participants was conducted once admitted for $1^{\text {st }}$ dose of RAI after thyroidectomy by the primary investigator. Informed consent with explanation of the risks and benefits as well as answering of other questions or concerns was done by the primary investigator in the respective rooms prior to RAI treatment.

\section{RESULTS}

A total of 52 participants were included in the study (Figure 1). Table 1 describes the baseline characteristics of the participants included in this study. The baseline characteristics between the two groups were similar. The median age of the participants in the ABW group (48.0) did not differ with those in the LBM group (48.0). Majority of participants were female $(n=49)$. The median BMI and LBM of the participants in the ABW group $\left(26.0 \mathrm{~kg} / \mathrm{m}^{2}\right.$ for BMI and $38.6 \mathrm{~kg}$ for LBM) did not differ with those in the LBM group $\left(26.4 \mathrm{~kg} / \mathrm{m}^{2}\right.$ for BMI and $39.1 \mathrm{~kg}$ for LBM) (Table 1).

Table 1. Baseline characteristics of the study population

\begin{tabular}{lrrr}
\hline Characteristics & ABW $\mathbf{( n = 2 6 )}$ & \multicolumn{1}{c}{ LBM $(\mathbf{n}=\mathbf{2 6})$} & p-value \\
\hline Age $(\text { years })^{\star}$ & $47.6 \pm 9.5(48.0)$ & $45.4 \pm 11.0(48.0)$ & 0.420 \\
\hline Sex & $3(11.5)$ & $0(0.0)$ & 0.235 \\
Male & $23(88.5)$ & $26(100.0)$ & \\
Female & $1(3.8)$ & $0(0.0)$ & 0.761 \\
\hline BMI $\left(\mathrm{kg} / \mathrm{m}^{2}\right)^{*}$ & $6(23.1)$ & $5(19.2)$ & \\
Underweight & $3(11.5)$ & $4(15.4)$ & \\
Normal & $13(50.0)$ & $11(42.3)$ & \\
At risk for obesity & $3(11.5)$ & $6(23.1)$ & \\
Obese I & $39.7 \pm 7.3(38.6)$ & $39.9 \pm 4.7(39.1)$ & 0.522 \\
Obese II & & &
\end{tabular}

Participants in the ABW group had significantly higher LT4 dosage $(2.2 \mathrm{mcg} / \mathrm{kg})$ compared to the LBM group $(1.4 \mathrm{mcg} / \mathrm{kg})(p$-value $<0.001)$ (Table 2). Participants in the ABW group had lower TSH levels at $6^{\text {th }}$ week $(5.7 \mathrm{uIU} /$ $\mathrm{mL})$ compared to the LBM group $(18.4 \mathrm{uIU} / \mathrm{mL})$ but this difference was not significant $(p$-value $=0.064)$. A significantly lower TSH level was observed at $12^{\text {th }}$ week in the ABW group $(1.6 \mathrm{uIU} / \mathrm{mL})$ compared to the LBM group $(3.8 \mathrm{uIU} / \mathrm{mL})(p$-value $=0.010)($ Table 2$)$.

Participants were divided based on risk stratification as low or intermediate risk for recurrence and or persistence of disease. Among the 52 participants, 25\% were intermediate risk and $75 \%$ were categorized low risk. 


\begin{tabular}{|c|c|c|c|}
\hline & $\begin{array}{c}\text { ABW }(n=26) \\
\text { Mean } \pm \text { SD } \\
\text { (median) }\end{array}$ & $\begin{array}{c}\text { LBM }(n=26) \\
\text { Mean } \pm S D \\
(\text { median) }\end{array}$ & $p$-value \\
\hline LT4 dosage $(\mathrm{mcg} / \mathrm{kg})$ & $2.2 \pm 0.1(2.2)$ & $1.4 \pm 0.1(1.4)$ & $<0.001$ \\
\hline TSH $6^{\text {th }}$ week (ulU/mL) & $5.7 \pm 9.3(1.4)$ & $18.4 \pm 28.5(4.5)$ & 0.064 \\
\hline TSH $12^{\text {th }}$ week (ulU/mL) & $1.6 \pm 2.5(0.4)$ & $3.8 \pm 5.5(2.2)$ & 0.010 \\
\hline
\end{tabular}

Table 3 shows the proportion of participants who achieved TSH suppression goal at $6^{\text {th }}$ and $12^{\text {th }}$ week. Nineteen percent of the participants (5 Low Risk, 0 Intermediate Risk) from ABW group reached TSH target at $6^{\text {th }}$ week while $26.9 \%$ (5 Low risk, 2 Intermediate Risk) from LBM group achieved TSH suppression goal. Forty-six percent (10 Low risk, 2 Intermediate risk) from ABW group and 50\% (10 Low risk, 3 Intermediate risk) from the LBM group achieved TSH target at $12^{\text {th }}$ week $(p$-value $=0.781)$.

Table 3. Comparison of participants who reached TSH target at $6^{\text {th }}$ and $12^{\text {th }}$ weeks

\begin{tabular}{lccc}
\hline & ABW $(\mathbf{n}=\mathbf{2 6})$ & LBM $(\mathbf{n}=\mathbf{2 6})$ & $\boldsymbol{p}$-value \\
\hline $6^{\text {th }}$ week & $5(19.2)$ & $7(26.9)$ & 0.510 \\
$12^{\text {th }}$ week & $12(46.2)$ & $13(50.0)$ & 0.781 \\
\hline
\end{tabular}

Table 4 shows the result of linear regression to determine the association between method of computation for LT4 dosage and the TSH levels at $6^{\text {th }}$ week $(\beta=-0.48,95 \%$ $\mathrm{CI}=-1.60$ to 0.63 , $p$-value $=0.382$ for $\mathrm{ABW}$ and $\beta=-0.17,95 \%$ $\mathrm{CI}=-0.38$ to 0.03 , $p$-value $=0.088$ for $\mathrm{LBM}$ ) and $12^{\text {th }}$ week ( $\beta=1.03,95 \% \mathrm{CI}=-3.77$ to 5.82 , $p$-value $=0.659$ for $\mathrm{ABW}$ and $\beta=-0.71,95 \% \mathrm{CI}=-1.76$ to 0.33 , $p$-value $=0.171$ for LBM).

Table 4. Association of LT4 dosage computed based on lean body mass and actual body weight with TSH level at $6^{\text {th }}$ and $12^{\text {th }}$ weeks

\begin{tabular}{cccc}
\hline & $\begin{array}{c}\text { Unadjusted } \\
\boldsymbol{\beta} \text {-coefficient }\end{array}$ & $\mathbf{9 5 \%} \mathbf{C l}$ & $\boldsymbol{p}$-value \\
\hline $6^{\text {th }}$ week & & & \\
ABW & -0.48 & -1.60 to 0.63 & 0.382 \\
LBM & -0.17 & -0.38 to 0.03 & 0.088 \\
\hline $12^{\text {th }}$ week & & & \\
ABW & 1.03 & -3.77 to 5.82 & 0.659 \\
LBM & -0.71 & -1.76 to 0.33 & 0.171 \\
\hline
\end{tabular}

Table 5 shows the result of logistic regression to determine the association between method of computation for LT4 dosage and the achievement of TSH suppression goal at $6^{\text {th }}$ and $12^{\text {th }}$ weeks; $(\mathrm{OR}=0.65,95 \% \mathrm{CI}=0.17$ to 2.38, $p$-value $=0.512$ and $\mathrm{OR}=0.86,95 \% \mathrm{CI}=0.29$ to 2.54 , $p$-value $=0.780$, respectively).

Table 5. Association of lean body mass versus actual body weight computation with achievement of TSH suppression goal at $6^{\text {th }}$ and $12^{\text {th }}$ weeks

\begin{tabular}{cccc}
\hline & $\begin{array}{c}\text { Unadjusted } \\
\text { Odds Ratio }\end{array}$ & $95 \% \mathrm{Cl}$ & p-value \\
\hline $6^{\text {th }}$ week & 0.65 & 0.17 to 2.38 & 0.512 \\
$\begin{array}{c}\text { ABW:LBM } \\
12^{\text {th }} \text { week } \\
\text { ABW:LBM }\end{array}$ & 0.86 & 0.29 to 2.54 & 0.781 \\
\hline
\end{tabular}

\section{DISCUSSION}

Different studies ${ }^{8,9}$ have suggested that LBM has been shown to be superior to other measures of body size for dosage of many drugs. ${ }^{8,9}$ LBM equals the total weight of a person's bone and muscles minus their fat weight. ${ }^{10}$ Pharmacokinetic parameters are mostly dependent on LBM and most metabolic processes occur within this body compartment.

Thyroid hormones are metabolized by different pathways (glucuronidation, sulfation and deiodination). Type 2 deiodinase enzyme which converts $\mathrm{T} 4$ to $\mathrm{T} 3$, has been detected in skeletal muscle, making this tissue an important site for T4 metabolism and degradation. ${ }^{8}$ Due to the importance of deiodination in the metabolism of thyroid hormones, studies have shown that LBM appears as the best correlate of LT4 daily requirements while fat mass has little or no effect. ${ }^{8}$ The observation of this study suggests that T4 metabolism mainly occurs within the lean compartment. ${ }^{8}$

To further understand the effect that body composition may exert on LT4, we studied a group of hypothyroid patients who underwent total thyroidectomy, received RAI ablation due to thyroid cancer and was placed on TSH suppressive doses. These patients were admitted for isolation and received RAI therapy at least 6 weeks after total thyroidectomy with RAI dose ranging from 50 to 150 mci. None of them were given recombinant TSH. The advantage of studying these group of patients ${ }^{8}$ were the following: no functioning thyroid tissue remnants, narrow TSH goals, and good compliance to drug prescriptions which is usually obtained for patients with cancer under strict follow up.

In this study, LBM was computed based on validated anthropometric prediction equations for estimation of LBM in Indian men and women ${ }^{6}$ in contrast to a few studies ${ }^{7-9}$ where LBM was measured by dual energy X-ray absorptiometry (DEXA). The use of this prediction equation was simple, reproducible, noninvasive, and inexpensive compared to the estimation of LBM by MRI or DEXA. The mean differences between the LBM measured by DEXA and prediction equation based on age, height, and weight in the case of men and women were $\sim 0.28 \mathrm{~kg}$ and $\sim 0.02 \mathrm{~kg}$, respectively with a standard error of estimate of $1.92 \mathrm{~kg} .{ }^{6}$

Among patients diagnosed with thyroid cancer, it is well established in literature that TSH levels must be suppressed to prevent recurrence or persistence of cancer. According to the 2014 ATA Guidelines for the Treatment of Hypothyroidism, ABW, TSH goal, ideal body weight, etiology of hypothyroidism, degree of serum TSH elevation, pregnancy, and age are factors that can influence dose requirement in patients with hypothyroidism. ${ }^{4}$ The current standard of care in the local setting, based on these guidelines, is to compute the initial LT4 dose based on ABW. In hypothyroid patients with minimal endogenous thyroid function, LT4 dose is computed at 1.6 to $1.8 \mathrm{mcg} / \mathrm{kg}$ ABW per day while in patients with thyroid cancer for suppression, the estimated doses are higher computed at 2 to $2.7 \mathrm{mcg} / \mathrm{kg} \mathrm{ABW}$ per day. ${ }^{4}$ Similar to the study of Olubowale and Chadwick, ${ }^{11}$ since the participants were all differentiated thyroid cancer patients, we computed both $\mathrm{ABW}$ and LBM at $2.2 \mathrm{mcg} / \mathrm{kg} /$ day. 
Our study revealed that the final LT4 dose was higher in the ABW group than in the LBM group likely because of the higher ABW than the computed LBM. LBM is made of body cell mass and the non-fatty intercellular connective tissue which would account for the significant difference in weight. From the analysis, we found that computation based on ABW required a mean LT4 dose of $2.2 \mathrm{mcg} / \mathrm{kg} /$ day compared with the LBM based computation which required a lesser dose of $1.4 \mathrm{mcg} /$ $\mathrm{kg} /$ day. With the anthropometric equation, the LT4 dose of LBM group was nearly $30 \mathrm{~kg}$ BW less than that of the $\mathrm{ABW}$ group. Hence, there was nearly one third less dose of LT4 in the LBM group.

Between the two groups, ABW was better than LBM in lowering the TSH near target levels. The TSH level of ABW at $6^{\text {th }}$ week was $5.7 \mathrm{uIU} / \mathrm{mL}$ compared to the LBM group $18.4 \mathrm{uIU} / \mathrm{mL}$ ( $p$-value $=0.064$ ) and this trend was consistent with the significant lower TSH level observed at $12^{\text {th }}$ week in the ABW group (1.6 uIU/mL) compared to the LBM group $(3.8 \mathrm{uIU} / \mathrm{mL})(p$-value $=0.010)($ Table 2$)$. This finding was similar to the study done by Sukumar et al., ${ }^{7}$ wherein mean TSH after 6 months revealed a lower TSH value seen using ABW than LBM (2.25 uIU/L vs $3.27 \mathrm{uIU} / \mathrm{L})$. In their study, they reported an increased requirement of LT4 with increasing weight as reported in literature. ${ }^{11}$ In addition, mean per $\mathrm{kg}$ body weight requirement of LT4 was higher in Asians compared to their western counterparts.

In this study, majority of the participants did not achieve TSH suppression goal by $6^{\text {th }}$ and $12^{\text {th }}$ weeks (Table 3 ). This is likely since according to literature, normalization of TSH following thyroidectomy can range from 4 to 6 months and dependent on the magnitude of change in dose from baseline. ${ }^{3}$

Our study results emphasize that the initial LT4 dose is more important in the achievement of desired TSH in the first weeks post RAI, before the first follow up and titration. In addition, statistical analysis suggests that LT4 dosage computed based on ABW or LBM was not significantly associated with TSH levels at $6^{\text {th }}$ and $12^{\text {th }}$ weeks. Furthermore, the odds of achieving TSH suppression goals at $6^{\text {th }}$ and $12^{\text {th }}$ weeks in patients who underwent total thyroidectomy and RAI ablation for differentiated thyroid cancer, were not significantly different between LT4 dosage computed using ABW and LBM ( $p$-value 0.512 and 0.781 , respectively) (Table 5). Hence, there is no significant difference between the two groups in terms of the effectiveness in achieving TSH suppression goal. This finding was in agreement to a previous study ${ }^{11}$ which showed that estimates of LBM based on routine anthropometric measurements were no better in predicting LT4 requirement in achieving $\mathrm{TSH}$ goals after total thyroidectomy than body weight. Moreover, another study even states that although total daily requirements of LT4 are related to LBM, there are unexplained differences such as ethnicity ${ }^{9}$ which occur among individuals of the same age and body size even in the absence of functioning thyroid tissue.

\section{CONCLUSION AND RECOMMENDATION}

Among patients with differentiated thyroid cancer who underwent $1^{\text {st }}$ time RAI therapy, ABW method of computation for LT4 dosage is better than LBM method due to the lower TSH trend seen at $6^{\text {th }}$ week and statistically significantly lower mean TSH at $12^{\text {th }}$ week, although, both method of computations did not achieve target TSH levels at $6^{\text {th }}$ nor $12^{\text {th }}$ week.

Since achievement of TSH target is not yet expected at $6^{\text {th }}$ nor $12^{\text {th }}$ week, we recommend a longer study duration to monitor TSH levels at 12 to 24 months after RAI ablation therapy with more patients of different weight categories and different target levels.

\section{Limitations}

A number of factors limited this analysis. For practicability, simplicity, convenience and desire to avoid cost, we employed a validated anthropometric prediction equation for estimation of LBM similar to the body composition of the study population which has a standard error of estimate of $1.92 \mathrm{~kg}$. Another limitation of this study was that baseline TSH prior to starting LT4 dosage was not documented. Although the participants were called every week to remind them about compliance and the same name brand of LT4 was prescribed to the participants, no pill count was done to confirm that they indeed took the medication with the same brand. Additionally, majority of thyroidectomy patients in our institution were prescribed with calcium supplements raising the concern regarding interference with LT4 absorption. Although participants have been educated to take them separately, compliance cannot be proven. Still, another limitation is the low achieved power of the logistic regression analysis computed at $14.4 \%$.

\section{Acknowledgments}

The authors would like to thank the staff and colleagues from St. Luke's Medical Center Quezon City, Section of Endocrinology, Diabetes and Metabolism for sharing their expertise and time in the assistance of the research.

\section{Statement of Authorship}

All authors certified fulfillment of ICMJE authorship criteria.

\section{Author Disclosure}

The authors declared no conflict of interest.

\section{Funding Source}

None.

\section{References}

1. Baehr KM, Lyden E, Treude K, Erickson J, Goldner W Levothyroxine dose following thyroidectomy is affected by more than just body weight. Laryngoscope. 2012;122(4):834-8. PMID: 22374624. https://doi. org/10.1002/lary.23186.

2. Burmeister LA, Goumaz MO, Mariash $\mathrm{CN}$, Oppenheimer $\mathrm{JH}$. Levothyroxine dose requirements for thyrotropin suppression in the treatment of differentiated thyroid cancer. J Clin Endocrinol Metabol. 1992;75(2):344-50. PMID: 1639933. https://doi.org/10.1210/ jcem.75.2.1639933

3. Jin J, Allemang MT, McHenry CR. Levothyroxine replacement dosage determination after thyroidectomy. Am J Surg. 2013;205(3):360 3; discussion 363-4. PMID: 23369308. https://doi.org/10.1016/j. amjsurg.2012.10.015

4. Jonklaas J, Bianco AC, Bauer AJ, et al. Guidelines for the treatment of hypothyroidism: Prepared by the American Thyroid Association task force on thyroid hormone replacement. Thyroid. 2014;24(12):1670 751. PMID: 25266247. PMCID: PMC4267409. https://doiorg/10.1089/ thy.2014.0028.

5. Cunningham JJ, Barzel US. Lean body mass is a predictor of the daily requirement for thyroid hormone in older men and women. J Am Geriatr Soc. 1984;32(3):204-7. PMID: 6699335. https://doi. org/10.1111/j.1532-5415.1984.tb02003.x. 
6. Kulkarni B, Kuper H, Taylor A, et al. Development and validation of anthropometric prediction equations for estimation of lean body mass and appendicular lean soft tissue in Indian men and women. J Appl Physiol (1985). 2013;115(8):1156-62. PMID: 23950165. PMCID: PMC3798815. https://doi.org/10.1152/japplphysiol.00777.2013.

7. Sukumar R, Agarwal A, Gupta S, et al. Prediction of LT4 replacement dose to achieve euthyroidism in subjects undergoing total thyroidectomy for benign thyroid disorders. World J Surg. 2010;34(3):527-31. PMID: 20044749. https://doi.org/10.1007/s00268-0090345-3.

8. Santini F, Pinchera A, Marsili A, et al. Lean body mass is a major determinant of levothyroxine dosage in the treatment of thyroid diseases. J Clin Endocrinol Metab. 2005;90(1)124-7. PMID: 15483074. https://doi.org/10.1210/jc.2004-1306.
9. Begum F, Ahmed CM, Afroz S, et al. Lean body mass-based levothyroxine replacement in young athyrotic patients with differentiated carcinoma of thyroid. Indian J Endocrinol Metab. 2013;17(2):254-9. PMID: 23776898. PMCID: PMC3683200. https://doi.org/10.4103/2230-8210.109697.

10. Roubenoff $\mathrm{R}$, Kehayias JJ. The meaning and measurement of lean body mass. Nutr Rev. 1991;49(6):163-75. PMID: 2046978. https://doi. org/10.1111/j.1753-4887.1991.tb03013.x.

11. Olubowale O, Chadwick DR. Optimization of thyroxine replacement therapy after total or near-total thyroidectomy for benign thyroid disease. Br J Surg. 2006;93(1):57-60. PMID: 16323163. https://doi. org/10.1002/bjs.5157.

Authors are required to accomplish, sign and submit scanned copies of the JAFES Author Form consisting of: (1) Authorship Certification, that authors contributed substantially to the work, that the manuscript has been read and approved by all authors, and that the requirements for authorship have been met by each author; (2) the Author Declaration, that the article represents original material that is not being considered for publication or has not been published or accepted for publication elsewhere, that the article does not infringe or violate any copyrights or intellectual property rights, and that no references have been made to predatory/ suspected predatory journals; (3) the Author Contribution Disclosure, which lists the specific contributions of authors; and (4) the Author Publishing Agreement which retains author copyright, grants publishing and distribution rights to JAFES, and allows JAFES to apply and enforce an Attribution-Non-Commercial Creative Commons user license. Authors are also required to accomplish, sign, and submit the signed ICMJE form for Disclosure of Potential Conflicts of Interest. For original articles, authors are required to submit a scanned copy of the Ethics Review Approval of their research as well as registration in trial registries as appropriate. For manuscripts reporting data from studies involving animals, authors are required to submit a scanned copy of the Institutional Animal Care and Use Committee approval. For Case Reports or Series, and Images in Endocrinology, consent forms, are required for the publication of information about patients; otherwise, appropriate ethical clearance has been obtained from the institutional review board. Articles and any other material published in the JAFES represent the work of the author(s) and should not be construed to reflect the opinions of the Editors or the Publisher.

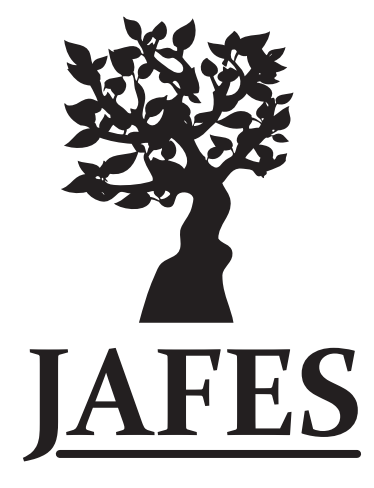

\section{A new venue for publishing your original articles. Visit www.ASEAN-endocrinejournal.org for Instructions to Authors.}

